\title{
Using global magnetospheric models for simulation and interpretation of Swarm external field measurements
}

\author{
T. Moretto ${ }^{1 *}$, S. Vennerstrøm ${ }^{2}$, N. Olsen ${ }^{2}$, L. Rastätter $^{1}$, and J. Raeder ${ }^{3}$ \\ ${ }^{1}$ NASA Goddard Space Flight Center, Greenbelt, Maryland, U.S.A. \\ ${ }^{2}$ Danish National Space Center, Copenhagen, Denmark \\ ${ }^{3}$ Space Science Center, University of New Hampshire, Durham, New Hampshire, U.S.A.
}

(Received January 5, 2004; Revised September 19, 2005; Accepted September 29, 2005; Online published April 14, 2006)

\begin{abstract}
We have used a global model of the solar wind magnetosphere interaction to model the high latitude part of the external contributions to the geomagnetic field near the Earth. The model also provides corresponding values for the electric field. Geomagnetic quiet conditions were modeled to provide simulated external contributions relevant for internal field modeling. These have proven very valuable for the design and planning of the upcoming multi-satellite Swarm mission. In addition, a real event simulation was carried out for a moderately active time interval when observations from the Ørsted and CHAMP sattelites were available. Comparisons between the simulation results and the satellite observations for this event demonstrate the current level of validity of the global model. We find that the model reproduces quite well the region 1 current system and nightside region 2 currents whereas it consistently underestimates the dayside region 2 currents and overestimates the horizontal ionospheric closure currents in the dayside polar cap. Furthermore, with this example we illustrate the great benefit of utilizing the global model for the interpretation of Swarm external field observations and, likewise, the potential of using Swarm measuremnets to test and improve the global model.
\end{abstract}

Key words: Ionospheric currents, field-aligned currents, global magnetospheric simulation, low-altitude satellites.

\section{Introduction}

The geomagnetic activity that is measured in the nearEarth space environment is the combined effect of vast, complex, and continually changing electric current systems in the Earths magnetosphere and ionosphere. Accurate parameterization of these current systems as well as improved understanding of the physical processes that drive them constitute an important goal of the planned multi-satellite Swarm mission (Friis-Christensen et al., 2006). This is necessary for advancing the inclusion of the external fields in the internal field modeling and for improving the data selection and correction for this modeling. In addition, the external current systems are a key ingredient in the solar windmagnetosphere-ionosphere system the full understanding of which is currently one of the main objectives within the field of space plasma physics.

The wide-spread and dynamic nature of the external current systems means that it is necessary to invoke models when interpreting sparsely distributed observations such as measurements from a few near-Earth spacecraft as suggested for the Swarm mission. Previously, empirical statistical models were mainly used for this purpose. Prominent examples are the model of high-latitude ionospheric

\footnotetext{
*Now at Division of Atmospheric Sciences, National Science Foundation, Arlington, Virginia, U.S.A.

Copyright (C) The Society of Geomagnetism and Earth, Planetary and Space Sciences (SGEPSS); The Seismological Society of Japan; The Volcanological Society of Japan; The Geodetic Society of Japan; The Japanese Society for Planetary Sciences; TERRAPUB.
}

current distributions developed by Friis-Christensen et al. (1985); the model of high-latitude ionospheric electrical potential distributions developed by Weimer (1995, 2001), and the recent model of high latitude field-aligned currents derived from geomagnetic satellite observations by Papitashvili et al. (2002). Similarly, empirical models that describe the magnetic variation at non-polar latitudes, such as the quiet day $\mathrm{Sq}$ current system and the equatorial electrojet, exist (e.g., Sabaka et al., 2002). Selfconsistent, physics-based models for the various parts of the complex solar wind- magnetosphere-ionosphere system are currently fast emerging. Global Magneto-Hydro-Dynamic (MHD) simulations are first-principle physics models that provide a self-consistent description of the large-scale magnetospheric configuration and dynamics as well as basic magnetosphere-ionosphere coupling driven by input solar wind parameters. A number of such models exist at present (e.g. Raeder, 2003). For the high-latitude ionospheres they provide realistic, and self-consistent, distributions of the horizontal electric field and currents in the ionosphere as well as the field aligned currents that couple the high latitude ionosphere to the outer magnetosphere. The validation of the global simulation models by comparison with observations is a very important task that currently attracts a lot of attention (e.g. Raeder et al., 1997; Keller et al., 2002; Ohtani and Raeder, 2004; Korth et al., 2004 and many others).

This paper together with the two accompanying papers by Vennerstrøm et al. (2006) and Ritter and Lühr (2006), 
present the first results of utilizing a global MHD model for the interpretation and simulation of Swarm external field measurements. Three different simulations representing quiet and moderately disturbed geomagnetic conditions have been performed. Focus here is on the effect of the high latitude ionospheric currents and corresponding near-Earth field aligned currents from a real event simulation of a time interval with moderate geomagnetic activity. We show examples of comparisons between the simulation results and measurements from the Ørsted and CHAMP geomagnetic satellite missions for this event. These serve to demonstrate the current level of validity of the global model for simulating Swarm observations and to illustrate the potential for very fruitful collaborations between global MHD model developers and the Swarm mission.

The global simulation model is described in the following section. Then follows a description of how the resulting magnetic and electric fields were calculated on a global grid at satellite altitudes. Next, follows a presentation of the three simulations that were performed for the Swarm studies so far. The next section presents the comparison between the results for the real event simulation and observations from the Ørsted and CHAMP satellites. The final section gives a summary and discusses future plans.

\section{The Open Geospace General Circulation Model}

Realistic estimates of the currents in the ionosphere and magnetosphere responsible for the external magnetic field contributions at polar latitudes are provided for this study by the Open Geospace General Circulation Model (OpenGGCM) (e.g. Raeder et al., 1995; Raeder et al., 1998) that is run at the Community Coordinated Modeling Center (CCMC) at NASA Goddard Space Flight Center. The model solves the resistive MHD equations in the magnetosphere. The simulation was run on a grid $160 \times 60 \times 60$ in size, spanning from -255 Earth radii $\left(R_{E}\right)$ to $33 R_{E}$ in the GSE $X$ direction and from $-48 R_{E}$ to $48 R_{E}$ in the GSE Y and Z directions. The grid has finest resolution of $0.4 R_{E}$ close to the inner magnetospheric boundary. The model also includes a magnetosphere-ionosphere coupling module that not only maps the field-aligned current densities, $J_{\|}$, into the ionosphere and the potential back into the magnetosphere, but also computes electron precipitation parameters and the ionospheric Hall and Pedersen conductances using empirical relations in a self-consistent manner (Raeder et al., 1998). Field-aligned currents are calculated close to the inner boundary of the magnetospheric part of the simulation (at $4 R_{E}$ ) and are used as input to solve the ionospheric potential equation. The field-aligned currents are mapped from points in the magnetosphere $\left(r_{M}, \vartheta_{M}, \lambda\right)$ into points in the ionosphere $\left(r_{I}, \vartheta_{I}, \lambda\right)$ along dipole field lines:

$$
\begin{aligned}
J_{\|}\left(r_{I}, \vartheta_{I}, \lambda\right)= & J_{\|}\left(r_{M}, \vartheta_{M}, \lambda\right)\left(\frac{r_{M}}{r_{I}}\right)^{3} \\
& \times \sqrt{\frac{4-3 \sin ^{2} \vartheta_{I}}{4-3 \sin ^{2} \vartheta_{I}\left(r_{M} / r_{I}\right)}}
\end{aligned}
$$

with

$$
\sin \vartheta_{I}=\sin \vartheta_{M} \sqrt{\frac{r_{I}}{r_{M}}}
$$

The polar ionosphere is treated as a two-dimensional spherical shell, thus the ionospheric potential equation reads (e.g. Kelley, 1989; Raeder, 2003):

$$
\nabla \cdot \underline{\underline{\Sigma}} \cdot \nabla \Phi=-J_{\|} \sin I
$$

with the boundary condition for the electric potential: $\Phi=$ 0 at the magnetic equator. $\underline{\Sigma}$ is the ionospheric conductance (i.e., height integrated conductivity) tensor, given by:

$$
\begin{aligned}
& \underline{\underline{\Sigma}}=\left(\begin{array}{cc}
\Sigma_{\vartheta \vartheta} & \Sigma_{\vartheta \lambda} \\
\Sigma_{\vartheta \lambda} & -\Sigma_{\lambda \lambda}
\end{array}\right) ; \Sigma_{\vartheta \vartheta}=\frac{\Sigma_{P}}{\sin ^{2} I} ; \\
& \Sigma_{\vartheta \lambda}=\frac{\Sigma_{H}}{\sin I} ; \Sigma_{\lambda \lambda}=\Sigma_{P}
\end{aligned}
$$

where $\Sigma_{H}$ is the Hall conductance, $\Sigma_{P}$ is the Pedersen conductance, $\vartheta$ is magnetic co-latitude, $\lambda$ is the magnetic longitude and $I$ is the magnetic field inclination angle.

The ionospheric Hall and Pedersen conductances play a key role in determining the ionospheric electrodynamics of the model. In the implementation of the model used for this study, they are computed from empirical formulas. The conductances are proportional to the ionospheric electron density (mostly dominated by the E-region), which is mainly determined by solar EUV irradiance and precipitation of magnetospheric electrons. The contribution to the conductance from the former is reliably parameterized by the solar radio flux parameter, $F_{10.7}$, together with the solar zenith angle (Moen and Brekke, 1993). The contributions to the conductance from magnetospheric electron precipitation are parameterized by the energy flux and mean energy of the precipitating electrons (Robinson et al., 1987). For the diffuse precipitation (from pitch angle scattering of hot magnetospheric electrons) these are parameterized, in turn, by the magnetospheric electron temperature and density, which are approximated by the density and temperature values from the magnetospheric part of the simulation. Additional discrete electron precipitation (auroral electrons accelerated by field-aligned potential drops) is parameterized by the field-aligned current density through the Knight relation (Knight, 1972).

The open-GGCM is driven by solar wind plasma and magnetic field conditions specified at the upstream simulation box boundary at $33 R_{E}$. For the simulation of real events, the solar wind observations must be propagated from a solar wind monitor satellite to this input position. The Earth magnetic field is approximated by a dipole with fixed orientation during the entire simulation run. Standard outputs from the model simulation include the magnetospheric plasma parameters (density, plasma pressure, velocity, magnetic field, and current densities), the ionospheric parameters (electric potential, field-aligned currents, and Hall and Pedersen conductances), and the mean energy and energy flux for the electron precipitation.

Validation and verification of not only this but all of the existing global models is a huge and many-faceted undertaking, involving the assessment of the numerical as well as 
the physical assumptions and approximations in the model (Raeder, 2003). It is outside the scope of this paper to do full justice to the many past and ongoing efforts on this problem. For the ionospheric parameters that are the focus of this study, obvious objects for validation are the inherent limitations in the model provided by the electrostatic, thinsheet approximation of the ionosphere and the reliance on empirical formulae for the calculating the conductance. Recent efforts have focused on replacing the simple sheet approximation for the ionosphere with fully dynamical multifluid ionosphere-thermosphere models (e.g. Raeder et al., 2001; Ridley et al., 2003). Specifically, this eliminates the need for invoking the Robinson formula in calculating the conductance contribution from electron precipitation and includes the ionospheric dynamo (neutral winds) effect. Raeder et al. (2001) found that this can significantly change the conductance and improve the comparison with ground based observations of the auroral electrojets. Ridley et al. (2003) reported neutral wind effects on the fieldaligned currents in the ionosphere of the order of 10-20 percent, with the strongest effect observed immediately following a northward turning of the IMF. Efforts to go beyond the electrostatic magnetosphere-ionosphere coupling adopted in the current models (e.g. allowing for parallel electric fields) are also under way (Lotko, 2004). Validation and improvement projects like these remain an important ongoing effort within the global modeling community at present. Since implementing models to run at the CCMC, this is increasingly becoming a wider community effort as well, and the study presented here is a contribution to this larger effort.

\section{Simulated Swarm external magnetic and elec- tric field contributions}

To produce estimates of the Swarm external fields the standard outputs from the open-GGCM model must be augmented. First, the three-dimensional distribution of the field-aligned currents in the "gap" between the inner boundary of the magnetospheric part of the simulation (at $4 R_{E}$ ) and the ionosphere (at $90 \mathrm{~km}$ altitude) were calculated by mapping of the field-aligned current density as described by Eq. (1). This was done on a spherical grid that is equidistant in the angular coordinates $(\vartheta, \lambda)$ with 129 and 128 grid points, respectively, and has a distribution in the radial coordinate given by:

$$
r_{n}=r_{0}+\frac{r_{1}-r_{0}}{\exp (1)-1}\left[\exp \left(\frac{n^{2}}{\left(n_{r}-1\right)^{2}}\right)-1\right]
$$

where $r_{0}$ is the lower radius $\left(1.014 R_{E}\right), r_{1}$ is the upper radius $\left(4 R_{E}\right)$, and $n_{r}=110$ is the number of grid points in the radial direction. The horizontal ionospheric (sheath) current distribution is calculated (on the corresponding grid in $\vartheta$ and $\lambda$ ) from the ionospheric electric potential and conductances by:

$$
\begin{aligned}
\mathbf{j}_{\text {horiz }} & =\left(\begin{array}{c}
j_{\vartheta} \\
j_{\lambda}
\end{array}\right)=\underline{\underline{\Sigma}} \cdot(-\nabla \Phi) \\
& =\underline{\underline{\Sigma}} \cdot \mathbf{E}_{\text {horiz }}=\underline{\underline{\Sigma}} \cdot\left(\begin{array}{c}
E_{\vartheta} \\
E_{\lambda}
\end{array}\right)
\end{aligned}
$$

where $\mathbf{E}_{\text {horiz }}$ is the ionospheric electric field. Finally, the magnetospheric current distribution was calculated on the continuation of the spherical grid with equidistant spacing of $0.5 R_{E}$ in the radial component from $4.5 R_{E}$ out to $20 R_{E}$ (32 grid points). All together, these contributions provide the global three-dimensional current distribution from which the magnetic field measurements at Swarm altitudes were calculated.

For any given distribution of current density, $\mathbf{J}$, the corresponding magnetic field can be calculated directly by integration according to Biot-Savart's law (see Vennerstrøm et al., 2006) The main problem with this approach is that the computation becomes very time-consuming when the number of grid-points is large. The advantage of this direct approach, on the other hand, is that it allows for the comparison between the contributions from different parts of the total current distributions. Results from such an analysis are presented by Vennerstrøm et al. (2006).

A computationally very efficient technique for calculating the magnetic field due to a given current distribution was developed by Engels and Olsen (1998). The method is based on the decomposition of divergence free vector fields into poloidal and toroidal parts following Stern (1976) and Backus (1986). Assuming that the time and length scales of the current density and magnetic fields considered are such that displacement currents can be neglected, which is equivalent to a non-divergent current density $\operatorname{div} \mathbf{J}=0$, and using that $\operatorname{div} \mathbf{B}=0$ everywhere, it is possible to decompose both the current density $\mathbf{J}$ and the associated magnetic field $\mathbf{B}$ into poloidal and toroidal parts, both of which can be expressed by scalar fields. The toroidal part of the magnetic field is determined by the poloidal part of the current density and the poloidal part of the magnetic field is determined by the toroidal part of the current density, independently and by fairly simple relations.

In the implementation of the technique adopted for this work, spherical harmonic expansions of the toroidal and poloidal scalar functions of the current density (and hence of those of the magnetic field) of degree and order 60 have been used. The magnetic field vector components corresponding to each of the current density distributions produced by the global model have been calculated on a spherical grid identical to the one used for the current densities. This product provided the basis for the analysis presented in the accompanying paper by Ritter and Lühr (2006)

The three-dimensional distribution of the electric potential at Swarm satellite heights has been calculated by mapping of the ionospheric electric potential along magnetic field lines, with the assumption that these are equipotentials. This was done on a spherical grid from the model ionosphere (90 km altitude) out to $1700 \mathrm{~km}$ altitude and provides the input needed for the modeling of the electric field measurements at the Swarm satellites. This grid has the same number of points for the $\vartheta$ and $\lambda$ components and has 33 points in the radial direction (grid point spacing of $50 \mathrm{~km}$ ), to allow for accurate estimation of the derivatives to get the electric field components. 


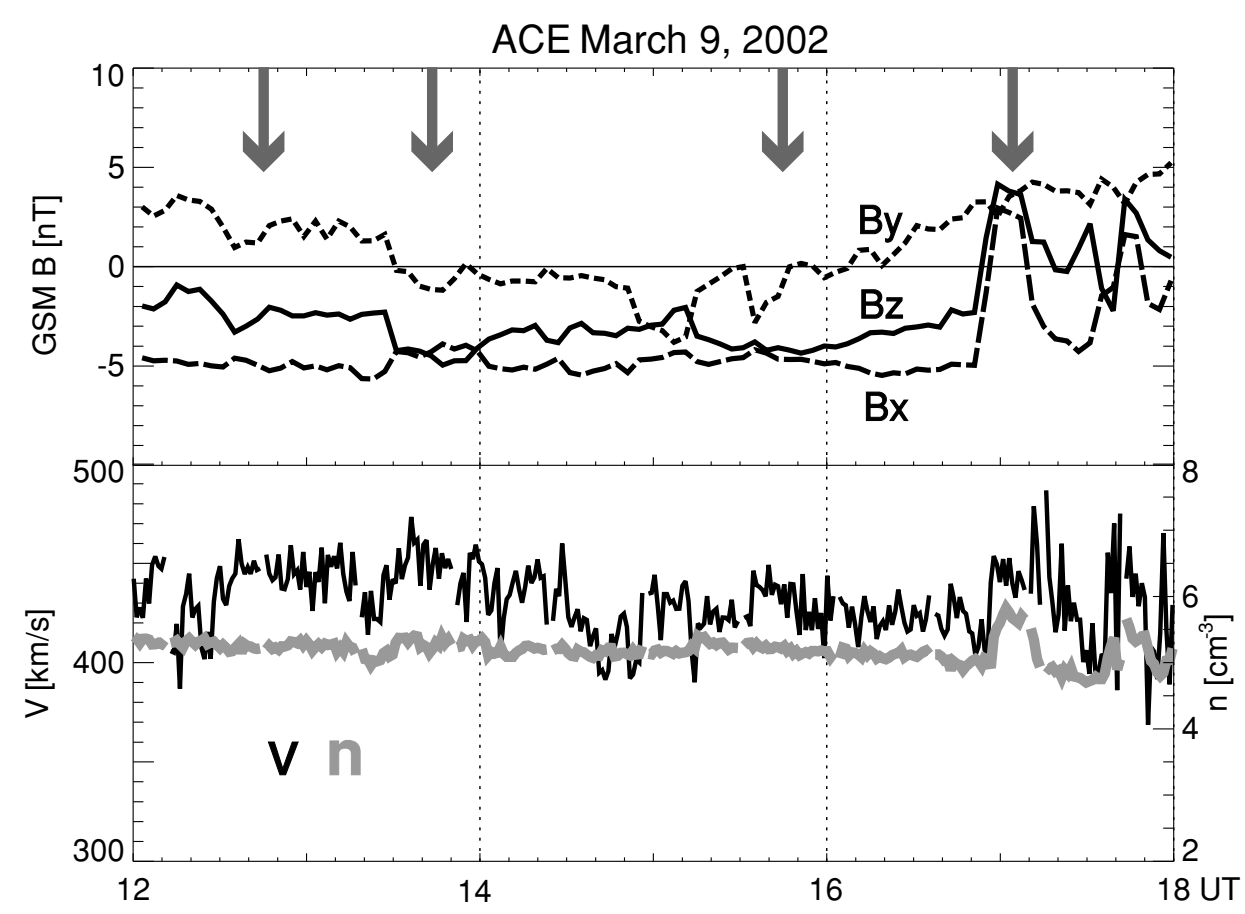

Fig. 1. Displayed are ACE observations of the interplanetary magnetic field and solar wind speed and number density that was used to drive the global magnetosphere model for March 9, 2002 12-18 UT. A delay of 55 min has been added to the spacecraft measurements to account for the solar wind propagation.

\section{Swarm Study Simulations}

While geomagnetic activity is driven by the combination of a wide variety of conditions in the solar wind and magnetosphere, including their time-history, the single most decisive parameter is the north-south component $\left(B_{Z}\right)$ of the interplanetary magnetic field (IMF). Keeping all other parameters at average values, a small, positive (northward) IMF $B_{Z}$ component generally will produce very low levels of geomagnetic activity. A small negative IMF $B_{Z}$ component, in contrast, is generally associated with moderate geomagnetic activity. Following the objective of the Swarm mission, simulations were first made for geomagnetic quiet and moderately disturbed conditions.

One simulation was driven by average density and solar wind values with the IMF $B_{Z}$ component changing slowly from a small positive to a small negative value over 8 hours. Specifically, the solar wind conditions used as input for the first simulation were: constant density, velocity, and temperature of $7 \mathrm{~cm}^{-3}, 400 \mathrm{~km} / \mathrm{s}$ (along the Sun-Earth axis), and $1.4 \times 10^{5} \mathrm{~K}$, respectively; IMF with zero $B_{Y}$ and $B_{X}$ GSM components and $B_{Z}$ component that changes linearly from $+5.5 \mathrm{nT}$ to $-5.5 \mathrm{nT}$ over the 8 hours of the simulation. A standard value of $150 \times 10^{-22} \mathrm{~W} / \mathrm{m}^{2} / \mathrm{Hz}$, was used for the $F_{10.7}$ parameter. Dipole tilt was zero making the model output exactly symmetric between the Northern and Southern hemispheres. The results from this simulation have been used in the analyses presented in the two accompanying papers of Vennerstrøm et al. (2006) and Ritter and Lühr (2006). Another simulation was performed with the same solar wind driving conditions but applying a tilt of the dipole axis. This allows for comparative analyses of the results for winter and summer conditions at the two polar regions. A dipole tilt of -25 degrees in the $\mathrm{X}-\mathrm{Z}$ plane was included, simulating winter conditions for the Northern hemisphere and summer conditions for the Southern hemisphere.

Finally, a simulation driven by actual solar wind observations for a quiet to moderately active period was performed to allow for the comparison of the model results with magnetic field measurements from the Ørsted and CHAMP satellites. The time interval of March 9, 2002, 06-18 UT was chosen for the simulation. The simulations were performed as so-called runs on request and the modeling results from all of the runs are available at the CCMC website (http://ccmc.gsfc.nasa.gov), keyword: swarm. Output from the simulations have been saved every 10 minutes.

For the real event simulation, solar wind observations from the ACE spacecraft were propagated to the simulation inflow boundary at $33 R_{E}$ upstream of the Earth. Focus here is on the more active, second half of the real event interval (12-18 UT) and the solar wind parameters that were used as input for this part of the real event simulation are shown in Fig. 1. They exhibit fairly stable values for the number density and velocity of approximately $5 \mathrm{~cm}^{-3}$ and $430 \mathrm{~km} / \mathrm{s}$, respectively. Until the abrupt change just before $17 \mathrm{UT}$, the IMF $B_{Z}$ component is southward with values between $-2 \mathrm{nT}$ and $-5 \mathrm{nT}$. The IMF $B_{Y}$ component starts out with a small positive value, then changes to near zero and slightly negative, and finally ends up again with a significant positive value. We note that the IMF $B_{X}$ component has a value of $-5 \mathrm{nT}$ throughout most of the interval but that this was not included in the simulation. A dipole tilt appropriate for the date and time was applied and was updated every two hours of the run.

The main sources for the magnetic perturbations measured at the altitude of Swarm are the ionospheric horizontal 

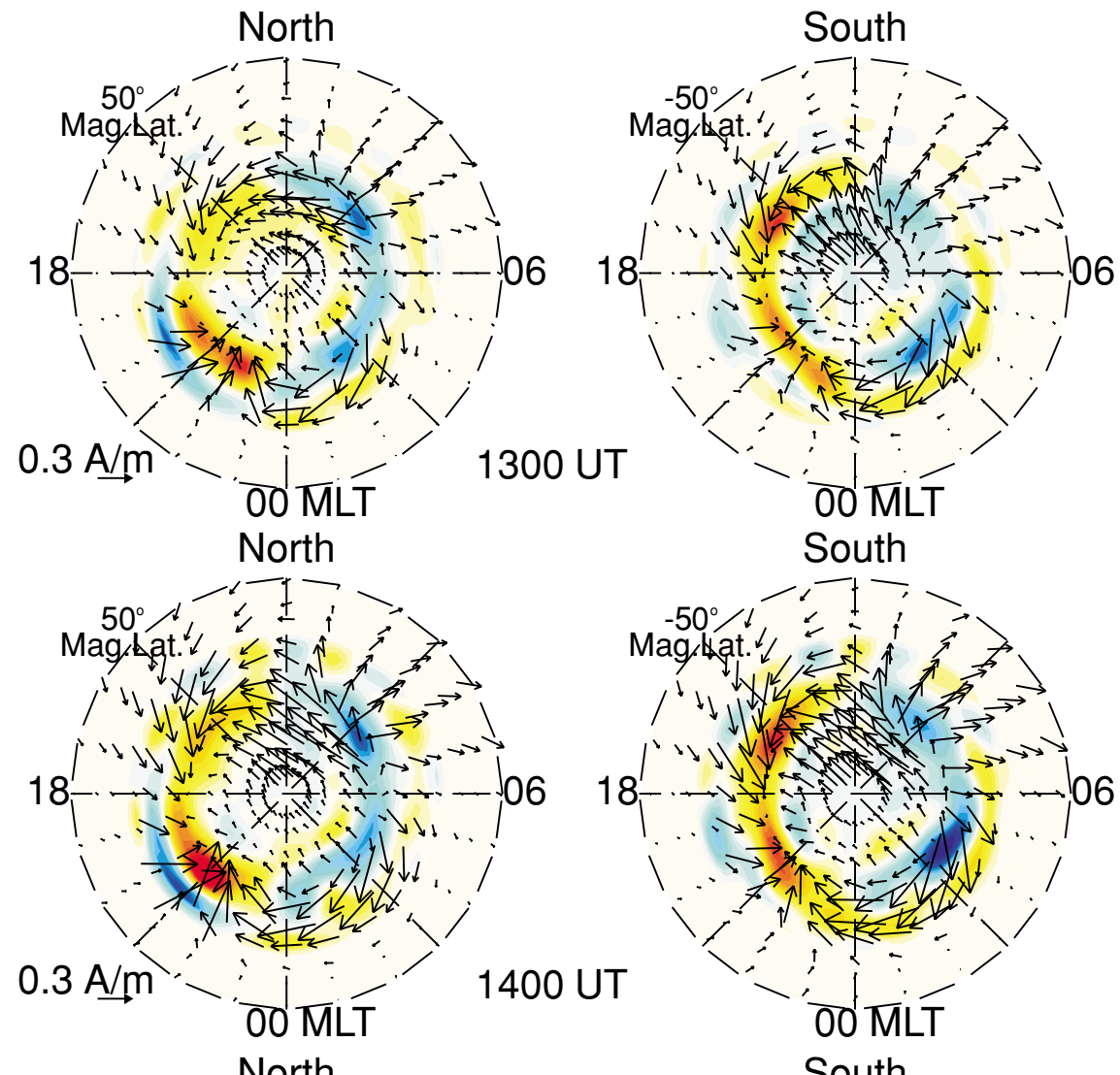

South
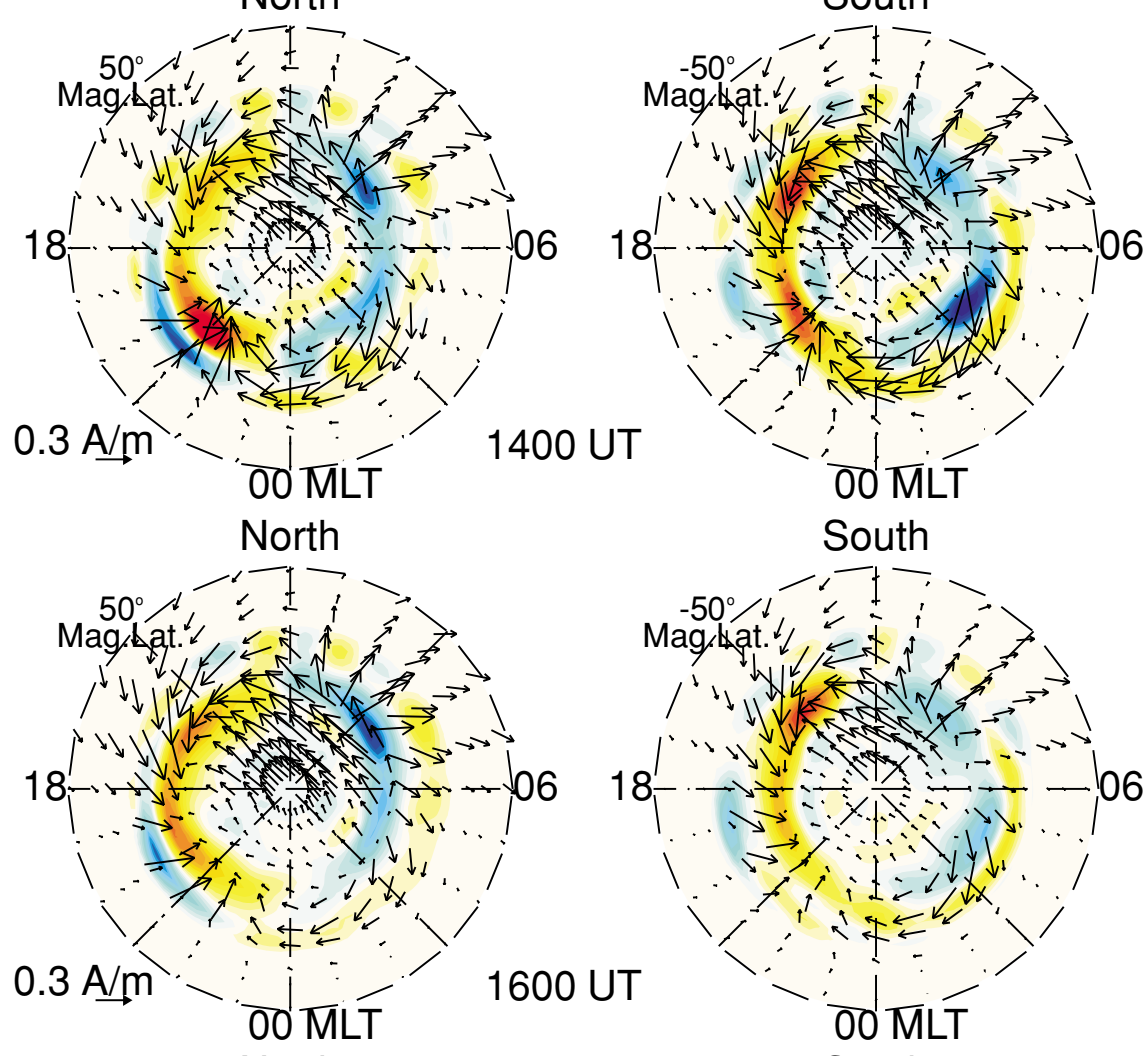

1400 UT
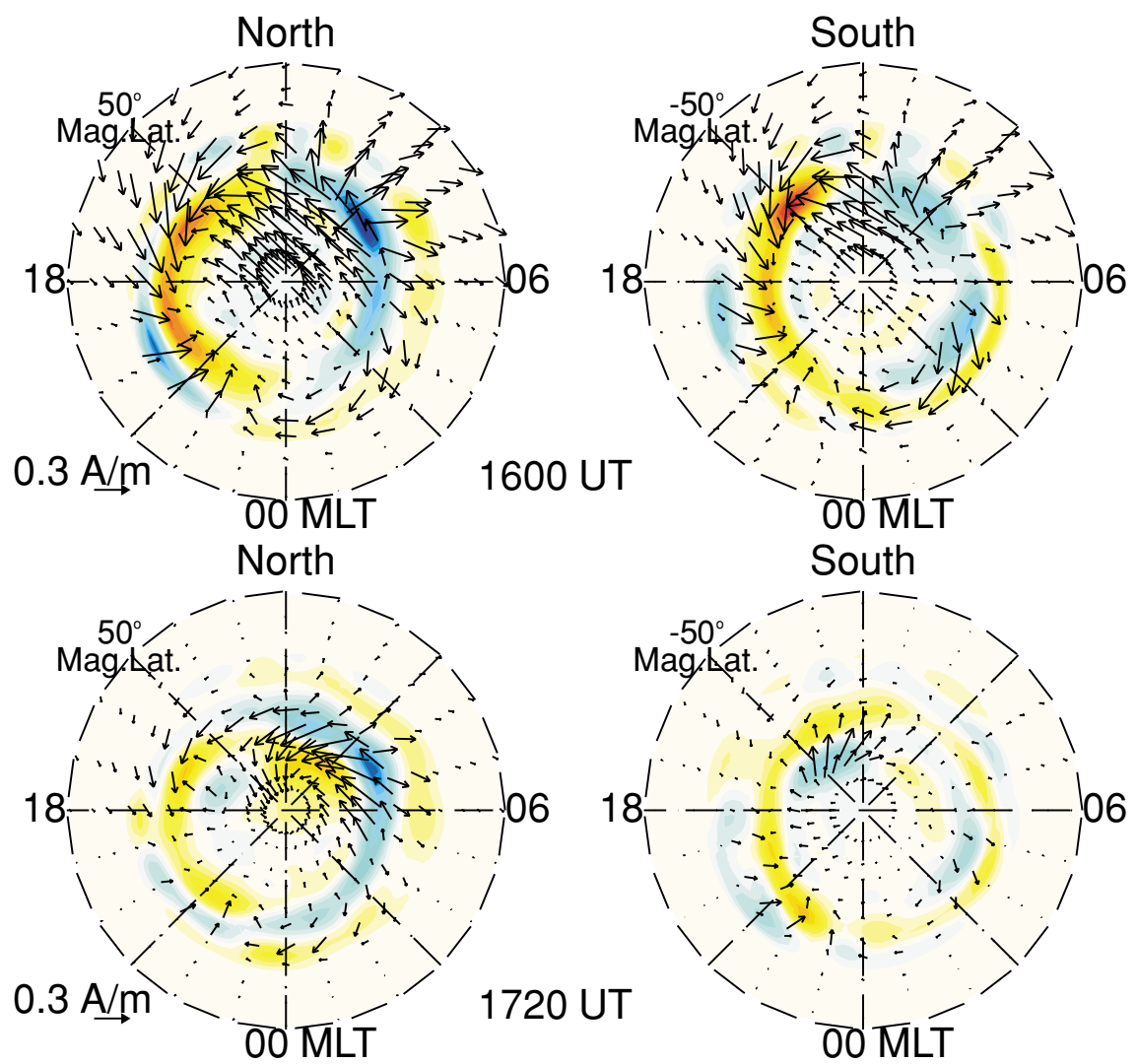

$-1.5$

0.0

$1.5 \mu \mathrm{Am}^{-2}$

$$
J_{\|}
$$

Fig. 2. Polar maps of the current densities from the open-GGCM simulation of March 9, 2002 12-18 UT are shown for both the northern (left) and southern (right) high latitude ionospheres $(90 \mathrm{~km}$ altitude) at four selected times. The color scale shows the field aligned current density distribution (blue for currents flowing into the ionosphere and yellow/red for currents flowing out). The horizontal ionospheric (sheet) currents are shown as black arrows. 
and field aligned currents, with only very small contributions from the distant magnetopause and tail currents (Vennerstrøm et al., 2006 (this issue)). Figure 2 displays polar maps of the distribution of these current sources over the high latitude ionosphere in both hemispheres at four different times during the simulation. The selected times are marked by the broad arrows in Fig. 1, including an additional 15 min delay to account for the time it takes for the solar wind to propagate through the magnetosheath and for any changes to the dayside currents to get established (e.g. Ruohoniemi et al., 2002).

The first pattern at 1300 UT shows well-defined region 1 currents, out of the ionosphere (yellow) on the dusk side and into the ionosphere (blue) on the morning side, roughly along the $75^{\circ}$ latitude circle. This is consistent with the IMF having a small negative $B_{Z}$-component at this time (Iijima and Potemra, 1976). An additional set of field aligned currents in the dayside polar caps (poleward of the region 1 currents) with opposite polarity in the two hemispheres (yellow in the northern and blue in the southern) are also observed. This is consistent with the significant positive $B_{Y^{-}}$ component that is present in the IMF at this time (FriisChristensen et al., 1985). This gives rise to a distinct asymmetry also for the horizontal currents on the dayside between the northern and southern hemisphere. At the time of the next set of plots at $1400 \mathrm{UT}$, the IMF $B_{Y}$-component is close to zero and, correspondingly, the polar cap currents are no longer present. As expected for this case, the current patterns for the two hemispheres are now very similar. They also have intensified slightly as a result of the increased southward IMF. At the time of the next set of maps at $1600 \mathrm{UT}$, the northern hemisphere map indicates fairly steady conditions for the dayside. Variations caused by dynamic changes in the tail magnetosphere (substorms) are seen in the nightside currents. The pattern for the southern hemisphere at this time is similar but the ionospheric current intensities in the polar cap region are lower. This is a result of reduced conductance in the southern dayside ionosphere as compared to the northern caused by the UTdependence of the dipole tilt. The dipole tilt is at its maximum ( $7^{\circ}$ in the $\mathrm{X}-\mathrm{Z}$ plane) around this time with the Northern magnetic pole pointing in the direction towards the sun. The last set of patterns at 1720 UT show much reduced current intensities both for the field aligned and horizontal currents reflecting the northward IMF conditions at this time. Also consistent with the northward IMF is the presence of a pair of currents of opposite polarity in the dayside of each polar cap. These are the so-called NBZ currents and the asymmetry displayed between the two currents of the pairs in both hemispheres as well as between the hemispheres is consistent with the influence of the significant positive $B_{Y^{-}}$ component in the IMF at this time (e.g. Vennerstrøm et al., 2002; Vennerstrøm et al., 2005).

\section{Comparison with Ørsted and CHAMP Mag- netic Field Observations}

On the date of the real event simulation (March 9, 2002), the orbit planes for the Ørsted (Neubert et al., 2001) and CHAMP (Reigber et al., 2002) satellites are fairly close. Between 12 UT and 14 UT, the satellites are moving nearly oppositely in their orbits so that they cross opposite poles almost simultaneously. We use the polar passes from two consecutive orbits during this period as our first illustration of the comparison between the simulation results and the magnetic field observations.

Figure 3 shows polar maps of the horizontal magnetic variation vectors (green arrows) together with the fieldaligned current distribution (background color scale) from the simulation for the two crossings over the northern polar region by Ørsted (left panels) and the corresponding south polar crossings by the CHAMP satellite (right panels). Matching the difference in the orbits, altitudes of $700 \mathrm{~km}$ and $450 \mathrm{~km}$ have been used for the maps for the Ørsted and CHAMP crossings, respectively. The observations are overlaid and are displayed as black arrows along the satellite tracks. The shown vectors are $5 \mathrm{sec}$ averages derived from $1 \mathrm{sec}$ vector magnetic field variation data from the satellites. The duration of the polar crossings is approximately 20 minutes and the time quoted for each map is the time when the satellite passes closest to the magnetic pole. The time in green at the bottom right of each panel is the time of the model output (green vectors and background color image).

Overall, the comparison in Fig. 3 shows remarkably good agreement between the model results and the satellite observations. In particular, the model seems to predict the strength and location of the region 1 currents (and their related magnetic variations) quite well. The main problem, on the other hand, seems to be the dayside polar cap area. This is especially clear for the CHAMP southern crossing at 1400 UT (lower right panel in Fig. 3). In this case, the model predicts rather strong and fairly uniform magnetic field vectors across the polar cap that do not match the CHAMP observations very well. The model magnetic field vectors reflect the presence in the model ionospheric current system at this time of strong horizontal currents over the dayside polar cap area as seen in Fig. 2 (second row). Our comparison therefore would indicate that these are not completely realistic. We speculate that this could result from the region 2 currents not being reproduced well enough in the simulation which then enforces too much closure of the region 1 currents across the polar cap. Some further evidence in support of this view is presented below. The magnetic effect of the horizontal currents decrease rapidly with altitude (Vennerstrøm et al., 2006) which is why the problem is more pronounced for CHAMP than for Ørsted.

Figure 4 displays in more detail the comparison between model results and observations along the satellite tracks for the four polar crossings of Fig. 3. The bottom row in each panel displays the horizontal magnetic variation vectors for the model (thick green arrows) and the observations (thin black arrows) along the satellite track. The only difference from the vectors in Fig. 3 is that the model vectors shown here have been calculated (by interpolation) directly along the satellite track. The traces at the top in each panel show the magnitude of the horizontal magnetic variations for the model (green line) and observations (black dots). Included in these plots are also the radial magnetic field perturbations (dashed green line for the model results and red dots for the satellite observations). As expected these are mostly of 

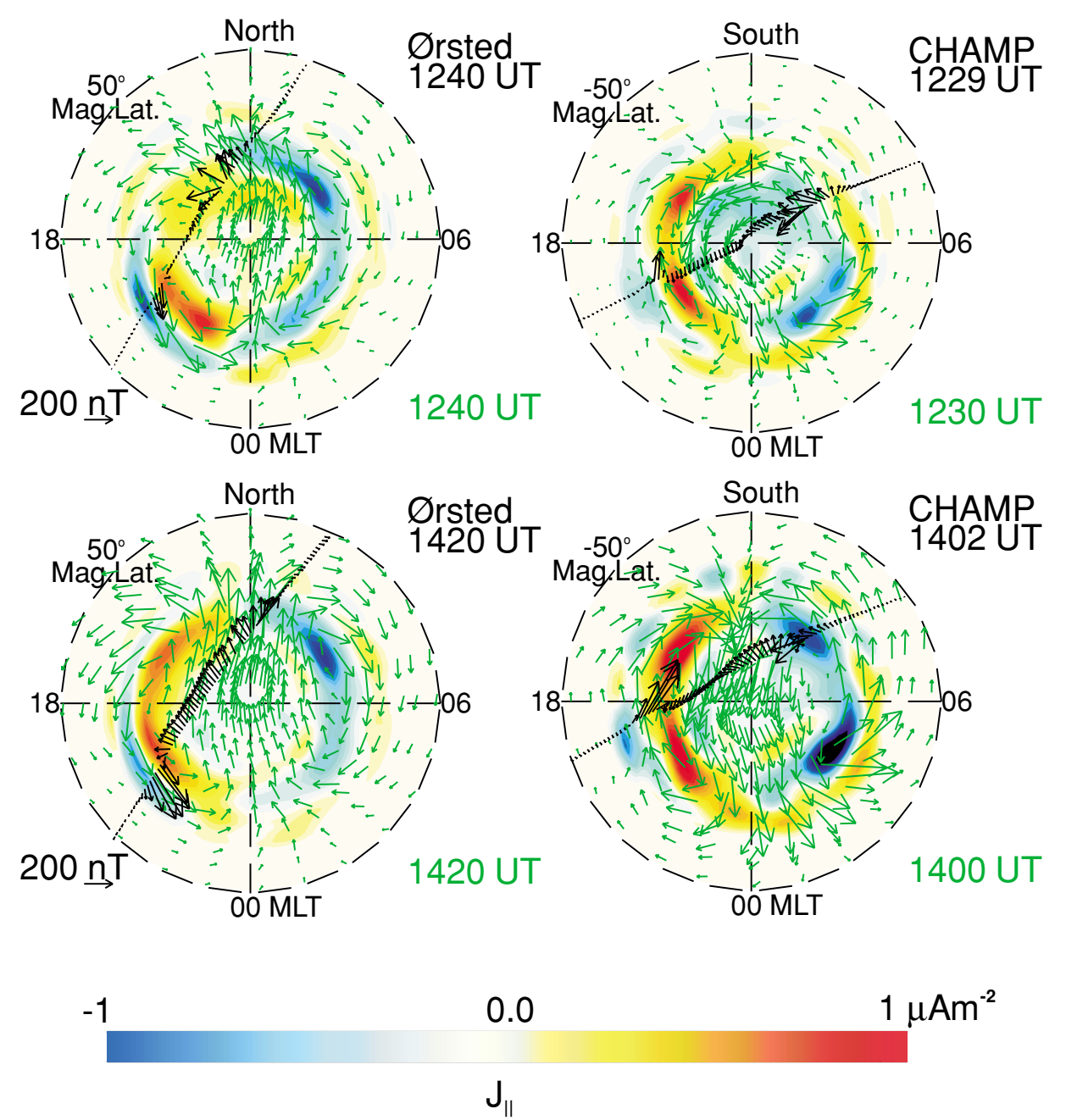

Fig. 3. Polar maps of the horizontal magnetic variation vectors derived from the open-GGCM simulation of March 9, 2002 12-18 UT and measured by Ørsted or CHAMP are shown for four selected polar crossings. The background color scale shows the field aligned current density distributions like in Fig. 2. The horizontal magnetic variations from the model are shown as green arrows and the satellite observations as black arrows.
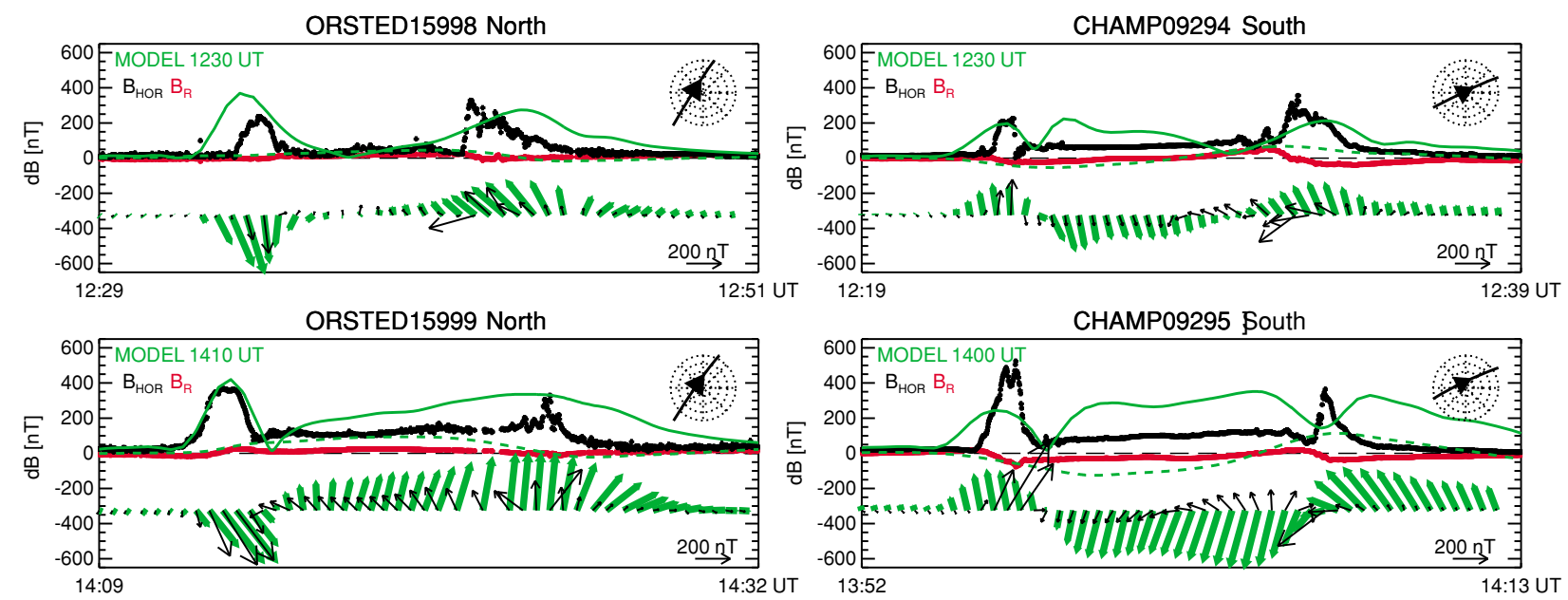

Fig. 4. Along track comparisons between model predictions and satellite observations of the horizontal and radial magnetic perturbations for the four polar crossings of Fig. 3. Model results are green arrows (horizontal magnetic vectors) and curves (magnitude of variation): dashed lines for the radial component and full lines for the horizontal component. Black and red dots in the upper tracks represent 5 sec averages of the magnitude of the horizontal and radial perturbations, respectively, measured by the satellites. Black arrows in the lower tracks display the measured horizontal magnetic variation vectors. 

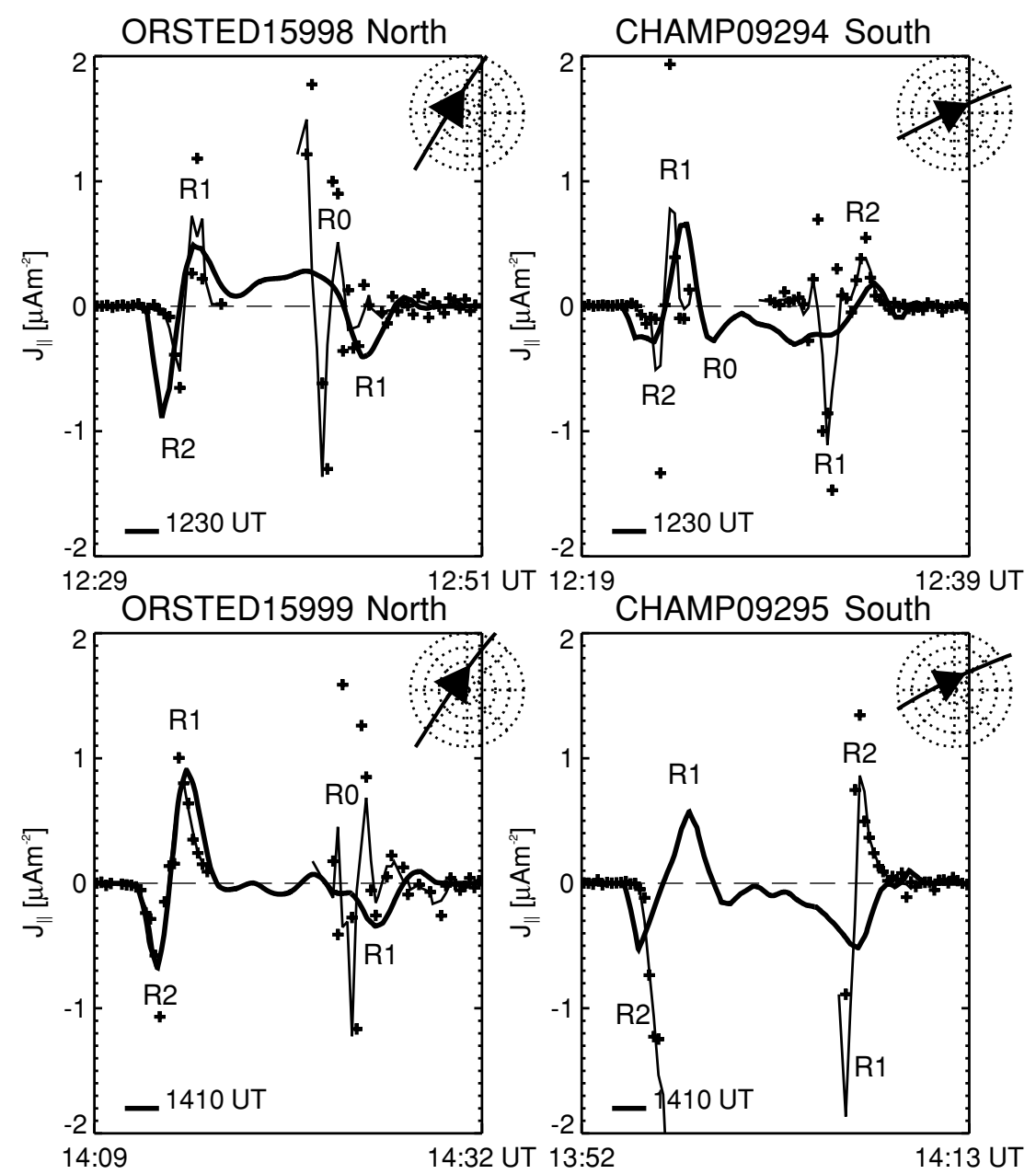

Fig. 5. Along track comparisons between model predictions and measurement based estimates of the field-aligned current density for the four polar crossings of Fig. 3. Model results are thick curves. Crosses represent $15 \mathrm{sec}$ averages of the current density estimates and the thin lines show $45 \mathrm{sec}$ averages. Negative values are for currents flowing into the ionosphere and positive values for currents flowing out.

much smaller magnitude than the horizontal perturbations (Vennerstrøm et al., 2006), their almost sole source being the horizontal ionospheric currents. This also means that we should not be surprised to find that they exhibit the same problem that we pointed out for the horizontal perturbations of sometimes overestimating the effect in the polar cap. The second southern CHAMP crossing (lower right panel in Figs. 3 and 4) is a prominent example of this.

For the horizontal variations, the single track comparisons largely confirm the findings that the polar maps in Fig. 3 convey. However, they also illustrate an inherent but important problem with using single space craft observations (or other sparse measurement sources) for testing of the model results. Namely, that even small displacements of structures, whose size; shape; and amplitude might otherwise be reproduced quite well by the model, show up as large discrepancies that may be misleading. This point is illustrated well by the nightside parts of the two Ørsted crossings (panels on the left in Figs. 3 and 4). The predicted nightside perturbations for the first crossing are much larger than observed (left part of upper left panel in Fig. 4), but from the total polar pattern in Fig. 3 (upper left panel) it is clear that the field-aligned current structure need only be rotated eastward by approximately $15^{\circ}$ to give a much im- proved match. In contrast, the nightside part of the following northward Ørsted crossing show a near perfect match (lower left panels in Figs. 3 and 4) between the predicted and observed perturbations. The field-aligned current structures in this case are much more sheet-like (wide-spread and near-homogeneous in longitude) and therefore are much less sensitive to the exact location of the structures in this direction relative to the satellite track. Part of this problem, of course, may be caused by inaccuracies in the mapping involved when deriving the satellite track, which is geographically defined, and the model which is defined in terms of a magnetic dipole system.

A complimentary view of the comparison is offered by the field-aligned currents. Adopting the infinite (east-west aligned) current sheet approximation for the field-aligned currents, a commonly used estimate for the field-aligned current density along the satellite track based on the magnetic field measurements (Ritter and Lühr, 2006) is given by:

$$
j_{\|}=\frac{d B_{\perp E}}{\mu_{0} \cdot d t \cdot v_{\perp} \cdot \cos \alpha}
$$

where $d B_{\perp E}$ is the difference between single measurements of the perpendicular magnetic field component in the local 

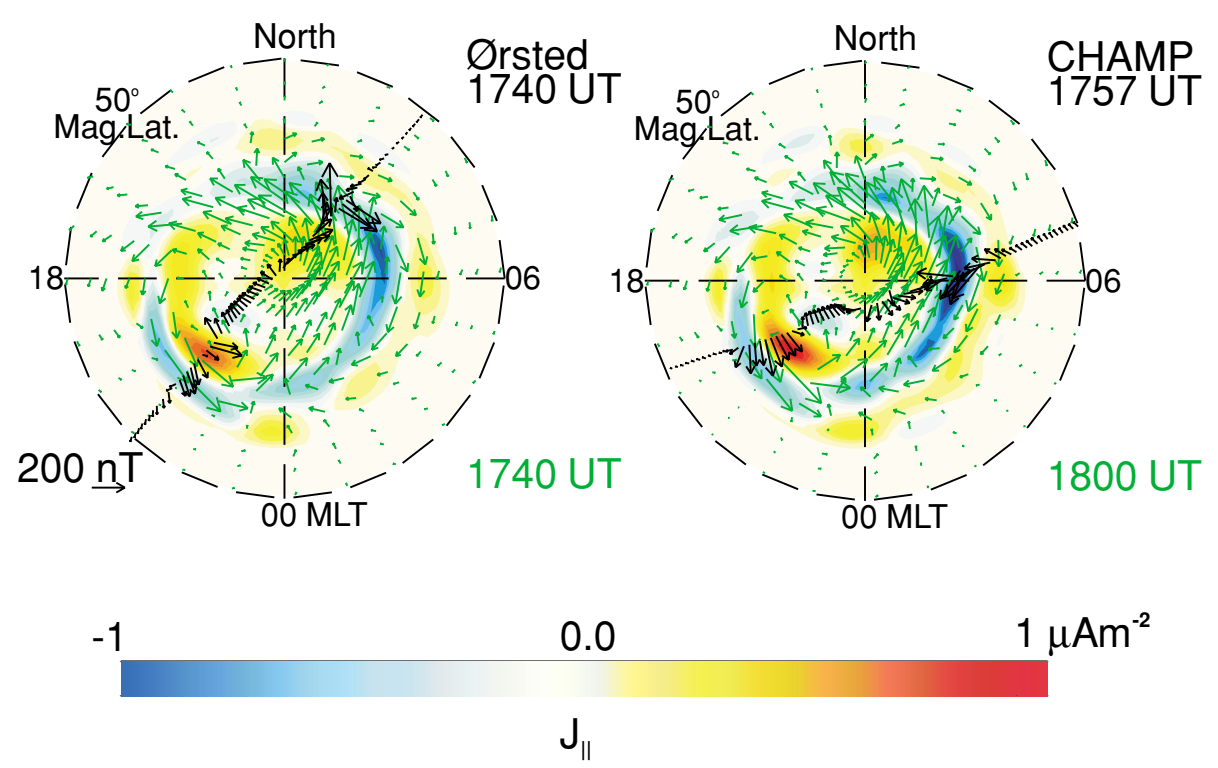

Fig. 6. Polar maps of the horizontal magnetic variation vectors derived from the open-GGCM simulation of March 9, 2002 12-18 UT and measured by Ørsted or CHAMP are shown for a two near-simultaneous North polar crossings. The background color scale shows the field aligned current density distributions like in Fig. 2. The horizontal magnetic variations from the model are shown as green arrows and the satellite observations as black arrows.
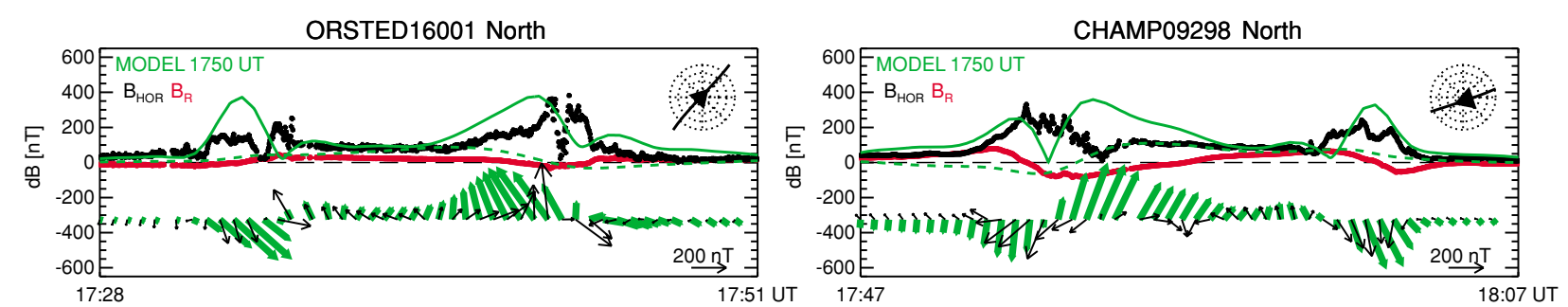

Fig. 7. Along track comparisons between model predictions and satellite observations of the horizontal and radial magnetic perturbations for the two polar crossings of Fig. 3. Model results are green arrows (horizontal magnetic vectors) and curves (magnitude of variation): dashed lines for the radial component and full lines for the horizontal component. Black and red dots in the upper tracks represent 5 sec averages of the magnitude of the horizontal and radial perturbations, respectively, measured by the satellites. Black arrows in the lower tracks display the measured horizontal magnetic variation vectors.

magnetic east direction (determined by the standard IGRF model); $d t$ is the time difference between measurements, here $1 \mathrm{sec} ; v_{\perp}$ is the projection of the satellite velocity into the plane perpendicular to the background magnetic field; and $\alpha$ is the angle of attack between the satellite track and the normal to the current sheet (assumed to be aligned in the local magnetic east-west direction). Figure 5 displays estimated current densities for the four polar crossings of Figs. 3 and 4 together with the corresponding field aligned current densities predicted by the global simulation. The latter are represented by thick black curves in the figure. The crosses represent $15 \mathrm{sec}$ averages of the calculated $1 \mathrm{sec}$ current density estimates and the thin lines represent $45 \mathrm{sec}$ running averages of the same. This corresponds to spatial resolutions of approximately $100 \mathrm{~km}$ and $300 \mathrm{~km}$, respectively. Estimates are only calculated for the parts of the satellite tracks that fulfill: $\alpha<60^{\circ}$. Where they could be identified with reasonable certainty, the main large-scale field-aligned current regions, Region 1, Region 2, and Region 0 (or Cusp and Polar Cap currents) have been called out in the panels as R1, R2, and R0, respectively. These re- sults confirm the generally good agreement between model predictions and measurements, particularly for the nightside parts of the crossings (to the left in each panel). The dayside estimated currents exhibit much more small-scale structure, which, of course, is not reproduced by the model. This is particularly evident when the spacecraft orbit passes near local noon on the dayside so that it goes through the Cusp region. The two Ørsted crossings (left panels in Figs. 3, 4, and 5) are clear examples of this. However, it is unlikely that the infinite-sheet approximation will hold well for these small-scale currents, which means that the measurement based estimates them-selves are likely to be flawed (Ritter and Lühr, 2006). The orbit of CHAMP does not pass as close to local noon as that of Ørsted and well-defined region 1 and region 2 currents on the dayside are observed in both of the CHAMP crossings (right hand panels in Figs. 3, 4, and 5). The comparison in Fig. 5 (right part of right hand panels) shows that for this case the model predicts region 1 currents of lower density than the estimates from the measurements but at the same time they are more spread out so that the integrated (total) current is about the 


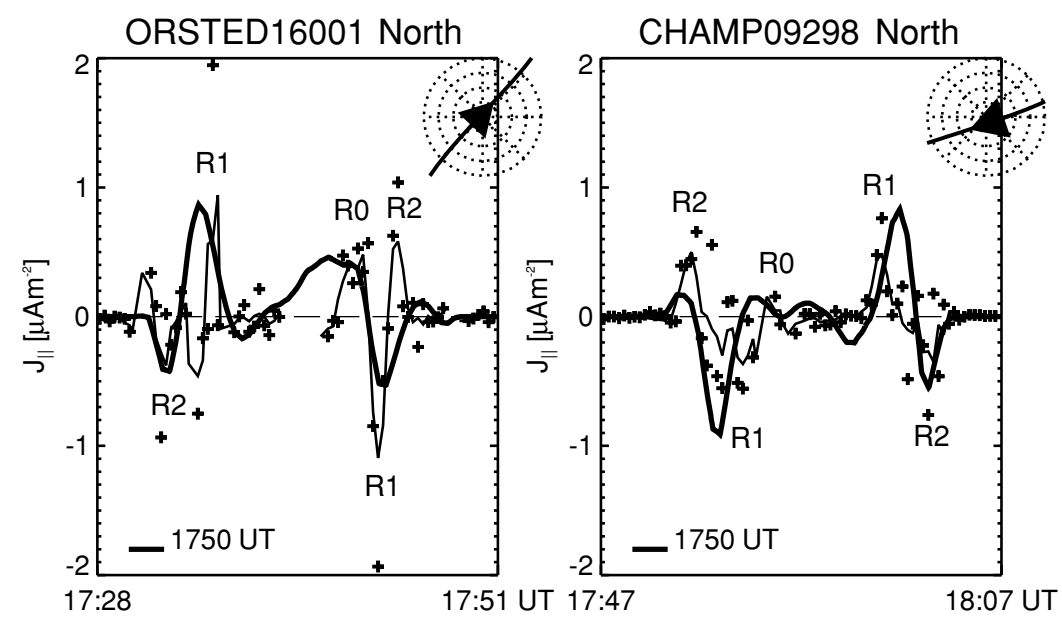

Fig. 8. Along track comparisons between model predictions and measurement based estimates of the field-aligned current density for the four polar crossings of Fig. 3. Model results are thick curves. Crosses represent $15 \mathrm{sec}$ averages of the current density estimates and the thin lines show $45 \mathrm{sec}$ averages. Negative values are for currents flowing into the ionosphere and positive values for currents flowing out.

same. In contrast, the region 2 currents seem to be consistently underestimated by the model. This tendency seems to get more pronounced as activity increases (from top row to bottom row of panels in the figure). These results support the conjecture made earlier that the problem with the simulation overestimating the dayside polar cap magnetic measurements during active conditions is mainly a result of lacking realistic region 2 currents in the model.

As a second example of the comparison between the global model results and measurements from Ørsted and CHAMP, we present the results for a pair of nearsimultaneous North-polar crossings close to the end of the simulation interval. In the same formats as earlier, Fig. 6 displays the polar maps, Fig. 7 the along-track perturbations, and Fig. 8 the field-aligned current densities. Note that now Ørsted crosses from the nightside to the dayside while CHAMP crosses in the opposite direction (from the dayside to the nightside). For this case, good agreement is found with the CHAMP observations on the nightside part of the crossing (right part of panel on the right) while the Ørsted observations (left part of panel on the left) indicate a more structured and complex current system than predicted. We believe that this is caused by Ørsted being very close to the Harang-discontinuity, the position of which the model may not have predicted accurately. For the dayside parts (right part of left hand panel and left part of right hand panel), both satellite measurements are reproduced quite well, though the region 2 current densities seem to be consistently underestimated as discussed for the first example.

\section{Summary and Outlook}

We have described a set of tools that we have developed to provide predictions for the swarm electric and external magnetic field measurements from global magnetohydrodynamic simulations of the solar wind interaction with the Earth's magnetosphere. Self-consistent sets of magnetospheric and ionospheric electric currents and corresponding electric and magnetic field perturbations at Swarm altitudes have been produced for several different geomag- netic activity conditions. These data sets were used in the analyses presented in the papers by Ritter and Lühr (2006) and Vennerstrøm et al. (2006).

For validation of our approach and to illustrate the potential of using the global MHD models for interpretation of the Swarm data, a simulation of a real time interval was performed using solar wind measurements to drive the model. These results were compared with magnetic field perturbation measurements from the Ørsted and CHAMP satellites for a number of polar crossings during the simulation interval. The comparison showed good agreement on many general, large-scale features between the model results and the satellite observations. In particular, the model seems to predict the strength and pattern of the region 1 currents (and their related magnetic variations) quite well. The most important discrepancies were found in the dayside polar cap region during more geomagnetically active conditions in which the model predictions greatly overestimated the measured magnetic perturbations, particularly for the lower altitude spacecraft (CHAMP). The large amplitude magnetic field perturbations in the model are caused by strong horizontal ionospheric currents flowing across the dayside polar cap providing closure for the dayside region 1 currents in the model. Hence, the problem is closely related to the weakness in the global MHD models of providing realistic region 2 currents. Our comparisons with the satellite observations show clear examples of the region 2 currents on the dayside being underestimated by the model.

It is a well-known limitation of the global MHD models at present that they do not include the ring current drift physics necessary to drive the region 2 currents in the magnetosphere. Although work is in progress to incorporate ring-current and inner magnetospheric convection models into the global MHD models (e.g. De Zeeuw et al., 2004), this has not so far been done in a self-consistent manner (i.e. including feed-back from the ring-current to the MHD model). It is obvious that the improved observational knowledge on the morphology and dynamics of the region 2 currents that the Swarm mission will provide will be a very valuable asset in this endeavor. 
The present study clearly verifies the value and mutual benefit of close collaboration between the global magnetospheric model developers and the Swarm mission. We have illustrated the unique capability of the global MHD models to provide global context for the Swarm measurements and to allow physics based interpretations of the observations. In turn, we have also demonstrated the unique capability of the Swarm mission to provide accurate tests of important model variables and, through that, valuable feedback to the model developers regarding the consistency and accuracy of the models. The development of realistic and precise predictive models is the main goal of current space weather research activities. Model validation through comparisons with observations is a very important component hereof. Another important objective is the development of methods to further constrain the models by assimilation of observational data. It is obvious that Swarm can make valuable contributions to both of these tasks. Immediately, in an obvious continuation of the efforts presented here and using observations from the current Ørsted and CHAMP missions as well as simulated future Swarm data a number of important questions can be addressed:

- What are the most important factors determining how well the model output match the measurements of magnetic fields and currents?

- How do we best define quantitative measures for the agreement to monitor improvements?

- How can the Swarm measurements be used in a systematic way to drive space weather prediction models?

- How can the Swarm observations best be used to further model development?

Acknowledgments. We gratefully acknowledge the use of the CHAMP vector magnetic field data courtesy of H. Lühr, GFZ Potsdam. The Ørsted Project was made possible by extensive support from the Ministry of Trade and Industry, the Ministry of Research and Information Technology, and the Ministry of Transport in Denmark. We thank the ACE SWEPAM and MAG instrument teams and the ACE Science Center for providing the ACE data. ESA (ESTEC) supported this study through contract No. 3-10901/03/NL/CB.

\section{References}

Backus, G., Poloidal and toroidal fields in geomagnetic field modeling, Rev. Geophys., 24, 75, 1986.

De Zeeuw, D. L., S. Sazykin, R. A. Wolf, T. I. Gombosi, A. J. Ridley, and G. Toth, Coupling of global MHD code and an inner magnetospheric model: Initial results, J. Geophys. Res., 109, A12219, doi:10.1029/2003JA010366, 2004.

Engels, U. and N. Olsen, Computation of magnetic fields within source regions of ionospheric and magnetospheric currents, J. Atm. Sol.-Terr. Physics, 60, 1585, 1998.

Friis-Christensen, E., H. Lühr, and G. Hulot, Swarm: A constellation to study the Earth's magnetic field, Earth Planets Space, 58, this issue, 351-358, 2006.

Friis-Christensen, E., Y. Kamide, A. D. Richmond, and S. Matshusita, Interplanetary magnetic field control of high-latitude electric fields and currents determined from Greenland magnetometer data, J. Geophys. Res., 90, 1325, 1985.

Iijima, T. and T. A. Potemra, The amplitude distribution of field-aligned currents at northern high latitudes observed by Triad, J. Geophys. Res., 81, 2165, 1976.

Keller, K. A., M. Hesse, M. Kuznetsova, L. Rastätter, T. Moretto, T. I. Gombosi, and D. L. DeZeeuw, Global MHD modeling of the impact of a solar wind pressure change, J. Geophys. Res., 107, 1126, 2002.

Kelley, M. C., The Earth's Ionosphere, Academic Press, New York, 1989.
Knight, S., Parallel electric fields, Planet. Space Sci., 21, 741, 1972.

Korth, H., B. J. Anderson, M. J. Wiltberger, J. G. Lyon, and P. C. Anderson, Intercomparison of ionospheric electrodynamics from the Iridium constellation with global MHD simulations J. Geophys. Res., 109, doi:10.1029/2004JA010428, 2004.

Lotko, W., Inductive magnetosphere-ionosphere coupling, J. Atm. Sol.Terr. Physics, 66, 1443, 2004.

Moen, J. and A. Brekke, The solar flux influence on quiet time conductances in the auroral ionosphere, Geophys. Res. Lett., 20, 971, 1993.

Neubert, T., M. Mandea, G. Hulot, R. von Frese, F. Primdahl, J. L. Jørgensen, E. Friis-Christensen, P. Stauning, N. Olsen, and T. Risbo, Ørsted satellite captures high-precision geomagnetic field data, EOS, 82, 81, 2001.

Ohtani, S.-I. and J. Raeder, Tail current surge: New insights from a global MHD simulation and comparison with satellite observations, J. Geophys. Res., 109, doi:10.1029/2002JA009750, 2004.

Papitashvili, V. O., F. Christiansen, and T. Neubert, A new model of field-aligned currents derived from high-precision satellite magnetic field data, Geophys. Res. Lett., 29, No. 14, doi:10.1029/2001GL014207, 2002.

Raeder, J., Global Geospace Modeling: Tutorial and review, in Space Plasma Simulations, edited by J. Buchner, C. T. Dunn, and M. Scholer, $\mathbf{6 1 5}$ of Lecture notes in physics, Springer Verlag, Berlin, 2003.

Raeder, J., R. J. Walker, and M. Ashour-Abdalla, The structure of the distant geomagnetic tail during long periods of northward IMF, Geophys. Res. Lett., 22, 349, 1995.

Raeder, J., J. Berchem, M. Ashour-Abdalla, L. A. Frank, W. R. Paterson, K. L. Ackerson, S. Kokubun, and J. A. Slavin, Boundary layer formation in the magnetotail: Geotail observations and comparisons with a global MHD simulation, Geophys. Res. Lett., 24, 951, 1997.

Raeder, J., J. Berchem, and M. Ashour-Abdalla, The geospace environment modeling grand challenge: Results from a global geospace circulation model, J. Geophys. Res., 103, 14787, 1998.

Raeder, J., Y. Wang, and T. Fuller-Rowell, Geomagnetic storm simulation with a coupled magnetosphere-ionosphere-thermosphere model, in Space Weather, AGU Geophys. Monogr. Ser., edited by P. Song, G. Siscoe, and H. J. Singer, Vol. 125, pp. 377, American Geophysical Union, 2001.

Reigber, C., H. Lühr, and P. Schwintzer, CHAMP Mission Status, Advances in Space Research, 30(2), 129, 2002.

Ridley, A. J., A. D. Richmond, T. I. Gombosi, D. L. De Zeeuw, and C. R. Clauer, Ionospheric control of the magnetospheric configuration: Thermospheric neutral winds, J. Geophys. Res., 108(A8), 1328, doi:10.1029/2002JA009464, 2003.

Ritter, P. and H. Lühr, Curl-B technique applied to Swarm constellation for determining field-aligned currents, Earth Planets Space, 58, this issue, 463-476, 2006.

Robinson, R. M., R. R. Vondrak, K. Miller, T. Dabbs, and D. Hardy, On calculating ionospheric conductances from the flux and energy of precipitating electrons, J. Geophys. Res., 92, 2565, 1987.

Ruohoniemi, J. M., S. G. Shepherd, and R. A. Greenwald, The response of the high-latitude ionosphere to IMF variations, J. Atm. Sol.-Terr. Physics, 64, 159, 2002.

Sabaka, T. J., N. Olsen, and R. A. Langel, A comprehensive model of the quiet-time near-Earth magnetic field: Phase 3, Geophys. J. Int., 151, 32, 2002.

Stern, D. P., Representation of magnetic fields in space, Rev. Geophys., 14, 199, 1976.

Vennerstrøm, S., T. Moretto, N. Olsen, E. Friis-Christensen, A. M. Stampe, and J. F. Watermann, Field-aligned currents in the dayside cusp and polar cap region during northward IMF, J. Geophys. Res., 107, doi:10.1029/2001JA009162, 2002.

Vennerstrøm, S., T. Moretto, L. Rastätter, and J. Raeder, Field-aligned currents during northward interplanetary magnetic field: Morphology and causes, J. Geophys. Res., 110(A6), doi:10.1029/2004JA010802, 2005.

Vennerstrom, S., T. Moretto, L. Rastätter, and J. Raeder, Modeling and analysis of solar wind generated contributions to the near-Earth magnetic field, Earth Planets Space, 58, this issue, 451-461, 2006.

Weimer, D. R., Models of high-latitude electric potentials derived with a least error fit of spherical harmonic coefficients, J. Geophys. Res., 100, 19,595, 1995.

Weimer, D. R., An improved model of ionospheric electric potentials including substorm perturbations and application to the GEM November 24, 1996 event, J. Geophys. Res., 106, 407, 2001.

T. Moretto (e-mail: tjorgens@nsf.gov), S. Vennerstrøm, N. Olsen, L. Rastätter, and J. Raeder 